\title{
El mito de la Andalucía subsidiada
}

CARMEN ANULA CASTELLS *

\begin{abstract}
En este ensayo se realiza un analisis preliminar de la información estadística disponible, y de algunas estimaciones realizadas por nosotros, al objeto de valorar la importancia real del gasto en prestaciones por desempleo en Andalucia, tanto en lo que se refiere al importe relativo de estos gastos en una perspectiva general como, especificamente, en lo que respecta al gasto en prestaciones por desempleo en el contexto del sector agrario andaluz. A partir de este análisis se concluye que carece de toda justificación atribuir a esta Comunidad Autónoma privilegio, ventaja o sesgo favorable alguno en la distribución de este tipo de gastos por las administraciones públicas del Estado.
\end{abstract}

Las prestaciones del desempleo agrario en Andalucía

Uno de los obstáculos más importantes en el análisis del nivel de subsidiación del desempleo en Andalucía no es sino la "opacidad" del INEM a la hora de ofrecer datos relativos a la magnitud de los subsidios del Régimen Especial Agrario de la Seguridad Social, lo que obstaculiza decisivamente la evaluación de la importancia relativa de dichos subsidios. No obstante, es posible realizar algunas estimaciones, a partir del número de parados perceptores de dichos subsidios, teniendo en cuenta los criterios de acceso por edades y la cuantía legal de las percepciones por desempleado (75\% del salario mínimo interprofesional). En el Cuadro 1 se muestra la estimación que hemos realizado para el año $1993^{1}$.

La estimación realizada del volumen total del subsidio agrícola andaluz correspondiente al año 1993 pone de manifiesto que, haciendo abstracción de la significación cualitativa del fenómeno, no es posible confirmar la existencia de una subsidiación que en términos cuantitativos pueda calificarse como

* Profesora de la Escuela de Relaciones Laborales de Sevilla.

1 El conjunto de los cuadros en que se apoya el análisis realizado en este trabajo se ofrecen, en forma de Apéndice Estadístico, al final del artículo. 
"masiva" de Andalucia, que tenga su raíz en las prestaciones de desempleo del Régimen Especial Agrario de la Seguridad Social (REASS).

El punto de partida del Cuadro 1 es el número de parados perceptores del subsidio del REASS por edades, recogido en la primera columna. Cada grupo de edad se hace corresponder con el número máximo de días de percepción a los que legalmente tienen derecho los trabajadores eventuales agrarios, siempre en el caso de que cumplan las condiciones exigidas de cotización, reflejado en la columna segunda del Cuadro 1.

La columna tercera del Cuadro 1 representa el montante del subsidio por día de prestación, equivalente al 75 por ciento del salario mínimo interprofesional (2.773 pesetas/día, en virtud del $R D$ 44/93, de 15 de enero de 1993). De esta forma, puede obtenerse el volumen anual estimado del subsidio por trabajador (cuarta columna del Cuadro 1), resultado de multiplicar, en cada caso, el número máximo de días de percepción anual por la cuantía legal de la prestación diaria. Finalmente, multiplicando el volumen estimado de las percepciones anuales por el número de perceptores se obtiene el total anual del subsidio de desempleo agrario para cada grupo de edad (quinta columna del Cuadro 1).

La estimación realizada pone de manifiesto que los 196.271 trabajadores 'eventuales agrarios andaluces que percibieron subsidios del REASS en ese año tuvieron derecho, en promedio, a 200 dias de percepción ${ }^{2}$, lo cual significó una prestación anual para cada trabajador equivalente, también en promedio, a 415.299 pesetas. Por tanto, la magnitud total efectiva del subsidio del REASS en Andalucía ascendió como máximo en 1993, según esta estimación, a 81.511 millones de pesetas.

De esta forma, podemos concluir, de acuerdo con estos cálculos, que el gasto total efectivo del subsidio de desempleo agrario andaluz representa, según la estimación realizada, algo más del 18 por ciento del gasto total en prestaciones por desempleo correspondientes a Andalucía en el año de referencia, como se deduce de las columnas sexta y séptima del Cuadro 1, lo cual no justifica las habituales caracterizaciones de una "Andalucía agraria subsidiada", y ello a pesar de la impresionante debilidad del mercado rural andaluz.

$2 \mathrm{Ha}$ de tenerse en cuenta que se han computado las cifras totales. Los trabajadores mayores de 52 años gozan de una cobertura superior a 180 días, de ahí que el promedio sea superior a los 180 días que marca la normativa. Este promedio de días de subsidio (así como el promedio de las percepciones anuales por este concepto) de trabajadores eventuales agrarios andaluces es obviamente superior al de la mayoría de los trabajadores: más del 78 por ciento de los mismos percibieron en este año únicamente 180 días de subsidio, lo que significa 374.355 pesetas al año como máximo. 
En efecto, como puede observarse en el Cuadro 2, la debilidad del mercado de trabajo rural andaluz en los siete últimos años (1987-1993) es inequívoca: la tasa de desempleo no ha sido en ningún caso inferior al 33 por ciento, mientras que ha llegado a superar la cota del 41 por ciento (en el año 1991). Por otra parte, la comparación de estas tasas con las correspondientes al conjunto del país revelan una clara asimetría: la tasa de desempleo agrario andaluz es entre 2,5 y 3 veces mayor que la tasa de desempleo agrario nacional (como refleja el índice de Desempleo del Cuadro 2, calculado sobre la base de igualar a 100 el volumen total del desempleo agrario en España), lo cual significa, si se tiene en cuenta que este último dato incluye las cifras andaluzas, que el desempleo agrario en el resto del país (excluido el de Andalucía) supone menos de la tercera parte del desempleo agrario andaluz para el período 1987-1993, siempre subre la base de las estadísticas de empleo del Instituto Nacional de Estadística (EPA).

\section{Las prestaciones del desempleo general en Andalucía}

Un análisis más detallado de las cifras relativas al gasto en prestaciones por desempleo pone de manifiesto, pese al importante volumen absoluto de prestaciones por desempleo en Andalucía, que ni los costes de prestaciones por trabajador desempleado ni las tasas de crecimiento de los costes de prestaciones por desempleo, tanto en términos relativos como absolutos, permiten deducir un sesgo favorable a esta región en el conjunto del sistema nacional de prestaciones. Los Cuadros 3, 4 y 5 muestran el reparto del gasto en prestaciones de desempleo por Comunidades Autónomas, en términos absolutos, en términos de crecimiento anual y en términos porcentuales, respectivamente.

De estos tres cuadros se deduce directamente que, en términos absolutos, Andalucía ha acaparado la mayor parte de los gastos en prestaciones, con una media para el período 1984-1993 del 21 por ciento (seguida de Cataluña y, a mayor distancia, por Madrid y Valencia), como se observa en el Cuadro 5 , lo cual no es más que un reflejo mecánico de los enormes diferenciales existentes entre Andalucía y el resto de Comunidades Autónomas en términos de número de desempleados. Sin embargo, las tasas de crecimiento de estos gastos revelan que no se ha producido sesgo alguno favorable a Andalucía, toda vez que la tasa media anual acumulativa se halla muy cercana a la nacional, como se desprende del Cuadro 4.

El panorama cambia de forma radical si, en lugar de considerar los costes totales, se consideran los costes unitarios del desempleo, es decir, los cos- 
tes medios anuales de prestaciones por trabajador desempleado. Los Cuadros 6, 7 y 8 recogen, respectivamente, el valor absoluto, las tasas de crecimiento y la estructura porcentual de dichos costes unitarios por comunidades autónomas.

Como se pone de manifiesto en los Cuadros 6 y 8 , los costes medios anuales del gasto de prestaciones por trabajador parado andaluz son inferiores a los costes medios anuales del gasto de prestaciones por trabajador parado español para todo el período considerado, 1984-1993, mientras que en regiones como Madrid, País Vasco, Cataluña o Navarra, la situación es exactamente la inversa.

Pero, además, si se tienen en cuenta las diferencias sectoriales, es posible afirmar, en base a nuestras estimaciones y a los datos ofrecidos en el Cuadro 6, que un obrero agrícola andaluz en paro percibió, en promedio, en el año 1993, menos de la tercera parte de lo recibido por un obrero catalán en la misma situación en concepto de prestaciones por desempleo, y menos de la mitad de lo percibido por un obrero madrileño, navarro, aragones o vasco, zonas éstas donde, como es sabido, se concentra buena parte de la industria nacional.

Finalmente, los datos relativos al crecimiento de los costes medios anuales del gasto en prestaciones por trabajador desempleado (Cuadro 8) perfilan aún más el carácter mítico de una Andalucía subsidiada masivamente: de acuerdo con la tasa media anual acumulativa calculada para el período 19841993, el crecimiento medio de estos costes es inferior en Andalucía (14,35\%) a la media nacional (15,2\%), lo cual es indicativo de un lento pero inexorable proceso de abaratamiento relativo de los costes unitarios del desempleo en Andalucía a lo largo de todo el período (no lo olvidemos) de vigencia del actual sistema que conjuga el PER y el subsidio del REASS (1984-1993). Ello contrasta con las cifras de crecimiento de Comunidades Autónomas como las de Murcia, Canarias, Madrid y, sobre todo, Cataluña (20,73\%).

El Cuadro 9 resume los resultados señalados, al mostrar el ranking (posiciones relativas) de las Comunidades Autónomas en los gastos de percepciones por desempleo en función de cuatro criterios: la magnitud de los costes totales (donde Andalucía aparece obviamente en primer lugar), el crecimiento de dichos costes totales y el volumen total y crecimiento de los costes unitarios. En los tres últimos casos Andalucía ocupa posiciones muy atrasádas, sobre todo teniendo en cuenta que la dinámica del mercado de trabajo andaluz (y específicamente el mercado de trabajo rural de esta región) no ha presentado un perfil de acercamiento con respecto a las cifras del mercado de trabajo del resto del país, manteniéndose por tanto las diferencias estructurales.

En efecto, la posición relativa de Andalucía de acuerdo con el criterio de crecimiento de los costes totales es la octava, por debajo de regiones más 
desarrolladas como Cataluña, Murcia o Valencia. Adicionalmente, si se tiene en cuenta tanto la magnitud de los costes unitarios como el crecimiento de los mismos, la situación empeora: en concreto, en lo que se refiere al crecimiento de los costes unitarios, Andalucía pasa a ocupar el undécimo lugar, detrás de Comunidades Autónomas como Madrid o Aragón, además de las ya mencionadas, de un nivel de desarrollo y renta per cápita mucho más elevados.

\section{Consideraciones finales}

Las cifras comentadas anteriormente desmienten con rotundidad el supuesto carácter desorbitado del volumen de las prestaciones del desempleo agrario andaluz, consideradas en su conjunto. Por otra parte, como hemos demostrado en otro lugar, dado que los ingresos derivados de las inversiones estatales realmente adscritas al Plan de Empleo Rural (PER) no poseen un carácter "extraordinario" con respecto a las inversiones locales realizadas en el resto de las Comunidades Autónomas del Estado español ${ }^{3}$, es difícil de justificar el constructo social que para este tipo de intervención estatal ha "diseñado" el discurso oficial, mistificador de la importancia real del régimen del subsidio de desempleo agrario y del PER, tanto desde los gobiernos central y autonómico, como desde las propias administraciones públicas y (al menos en parte) partidos políticos.

Valga como ilustración el significativo "cambio de opinión" (al menos, con respecto a la explicitada en el Congreso de los Diputados) de los grupos políticos que participaron en la Ponencia sobre la reforma del sistema: si la intención original de los grupos de la oposición parlamentaria (con la excepción del IU-IC) era la de reformar el sistema en el sentido de eliminar su carácter «fraudulento" y "privilegiado" con respecto a la intervención en otras Comunidades Autónomas (que, en su criterio, implicaba la "compra" del voto del campo andaluz a cambio del subsidio), las cifras globales sobre la importancia real del gasto público destinado a este sistema de intervención estatal acabaron por convencer a los señores diputados de lo injustificado del supuesto "privilegio"; como consecuencia, ninguno de los partidos de la oposición elevó propuestas significativas que pusieran en cuestión los fundamentos del

3 Para el año 1994, el gasto de inversión afectado realmente el PER (11.925,8 millones de pesetas) significó algo más del 11 por ciento del total de inversiones «teóricamente adscritas al Plan de Empleo Rural. Véase, en este sentido, Anula Castells, C.: El mercado, el Estado y la Familia. (Tesis Doctoral, no publicada) Sevilla, 1995; pág. 230. 
actual sistema, con la excepción del IU-IC, que insistía en la necesidad de reformas estructurales para atacar el problema del desempleo andaluz.

Por otra parte, las cifras relativas a los gastos generales de prestaciones por desempleo en Andalucía, que se han comentado más arriba, no avalan en absoluto las afirmaciones relativas a una extraordinaria subsidiación de los trabajadores desempleados en esta región. El mero análisis puntual de estas cifras pone claramente de manifiesto que ni la posición que ocupa la Comunidad Autónoma de Andalucía con respecto al resto de las regiones españolas, utilizando diversos criterios de clasificación sobre la base del volumen relativo de prestaciones por desempleo, ni en particular la evolución de los costes unitarios del gasto en prestaciones por desempleo, justifica calificar la situación de Andalucía como una situación de "privilegio»5.

\section{Anexo estadístico}

\section{Cuadro n..$^{\circ}$}

\section{Estimación del volumen de percepciones efectivas del subsidio agrario andaluz para el año 1993}

\begin{tabular}{|crrrrrrr|}
\hline Edad & $\begin{array}{c}\text { Número } \\
\text { perceptores }\end{array}$ & $\begin{array}{c}\text { Dias } \\
\text { percepción }\end{array}$ & $\begin{array}{c}\text { Percepción } \\
\text { dia (\#) }\end{array}$ & $\begin{array}{c}\text { Percepción } \\
\text { anual por } \\
\text { trabajador }\end{array}$ & $\begin{array}{c}\text { Total } \\
\text { SDA }\end{array}$ & $\begin{array}{c}\text { Total } \\
\text { GPD** }\end{array}$ & $\begin{array}{c}\text { Tasa } \\
\text { SDAJGPD }\end{array}$ \\
\hline$<25$ & 32511 & 180 & 2079.8 & 374355 & 12171 & - & - \\
25 a 51 & 121613 & 180 & 2079.8 & 374355 & 45526 & - & - \\
52 a 54 & 9145 & 240 & 2079.8 & 499140 & 4565 & - & - \\
55 a 59 & 21497 & 270 & 2079.8 & 561533 & 12071 & - & - \\
$>$ 60 & 11505 & 300 & 2079.8 & 623925 & 7178 & - & - \\
Todas las edades & $\mathbf{1 9 6 2 7 1}$ & $\mathbf{2 0 0}$ & $\mathbf{2 0 7 9 . 8}$ & $\mathbf{4 1 5 2 9 9}$ & $\mathbf{8 1 5 1 1}$ & $\mathbf{4 4 7 3 4 8}$ & $\mathbf{1 8 . 2 \%}$ \\
\hline
\end{tabular}

Fuente: Elaboración propia. Instituto Nacional de Empleo.

(\#) Corresponde al 75\% del salario minimo interprofesional diario (Real Decreto 44/93, de 15 de enero. 1993).

(*) Subsidio de desempleo agrario efectivo (gasto total anual), en millones de pesetas.

(**) Gasto en prestaciones por desempleo (gasto total anual), en millones de pesetas.

4 A este respecto véanse el informe elaborado por la ponencia especial para estudiar la reforma del actual sistema del Plan de Empleo Rural y el Subsidio Agrario y el Dictamen de la correspondiente comisión parlamentaria, en Boletin Oficial de las Cortes Generales, $\mathrm{n}^{\circ} 74$.

5 No obstante, la tasa de paro en Andalucía para el segundo trimestre de 1995, según estimaciones de la EPA, significa el 33,3\%, más de diez puntos porcentuales por encima de la tasa nacional. Véase INE: Encuesta de Población Activa. Principales resultados. Segundo Trimestre de 1995. INE, Madrid, 1995. 


\section{Cuadro 2}

Activos, ocupados y parados agrícolas (\#)

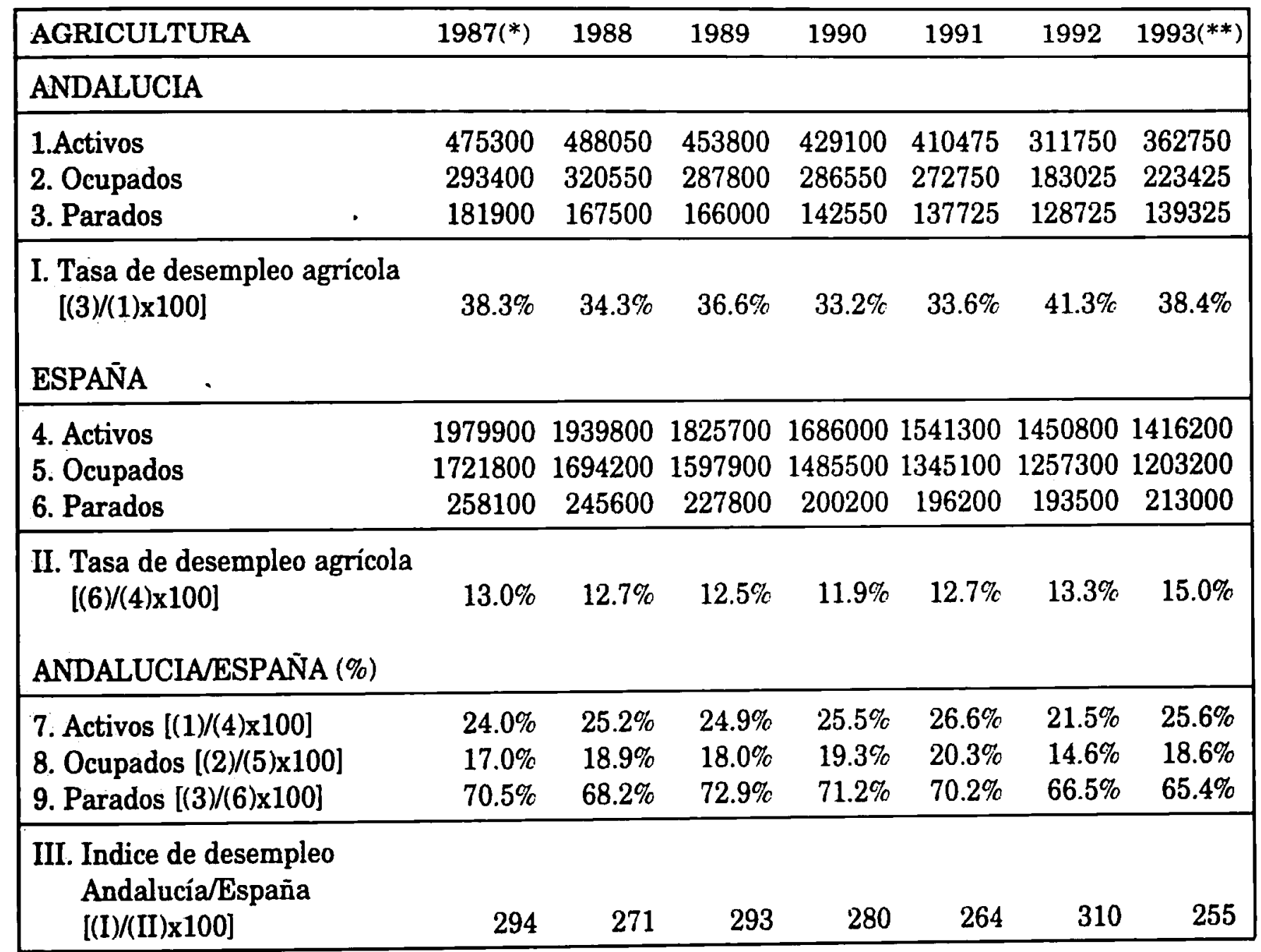

Fuente: Encuesta de Población Activa (INE)

(\#) Medias anuales.

(*) Excluido primer trimestre.

(**) Para los datos nacionales, excluido cuarto trimestre. 
Cuadro 3

Importe total de gastos de prestaciones por desempleo (millones de pesetas)

\begin{tabular}{|lrrrrrrrrrr|}
\hline Comunidades & 1984 & 1985 & 1986 & 1987 & 1988 & 1989 & 1990 & 1991 & 1992 & $1993\left(^{*}\right)$ \\
Autónomas & 118982 & 148882 & 169393 & 194491 & 215006 & 247630 & 296851 & 357383 & 415553 & 447348 \\
\hline Andalucia & 16837 & 20145 & 20620 & 20391 & 21776 & 23949 & 27677 & 37651 & 44061 & 51782 \\
Aragón & 17026 & 20686 & 21357 & 23471 & 25517 & 27828 & 31348 & 34046 & 40675 & 52017 \\
Asturias & 11237 & 14455 & 16655 & 18062 & 23064 & 27416 & 33561 & 39238 & 42919 & 41126 \\
Baleares & 14447 & 22609 & 25071 & 30051 & 36882 & 44836 & 60371 & 76660 & 83440 & 78356 \\
Canarias & 6479 & 8825 & 10111 & 11006 & 13016 & 13252 & 14572 & 18680 & 21571 & 26267 \\
Cantabria & 30440 & 33243 & 36193 & 39326 & 42679 & 46683 & 54759 & 68630 & 84447 & 91501 \\
Castilla León & 16376 & 21500 & 24926 & 27641 & 31194 & 37304 & 43173 & 53013 & 65916 & 72166 \\
Castilla La Mancha & 98316 & 137310 & 145604 & 146619 & 153890 & 171785 & 208983 & 269370 & 312504 & 383352 \\
Cataluña & 17624 & 20923 & 25215 & 27436 & 30412 & 33721 & 39188 & 44898 & 51009 & 53595 \\
Extremadura & 33283 & 49745 & 46900 & 51670 & 55912 & 63614 & 74414 & 92721 & 107934 & 117598 \\
Galicia & 73908 & 100047 & 103764 & 105121 & 109712 & 123674 & 145666 & 181465 & 227502 & 270872 \\
Madrid & 8821 & 12090 & 13278 & 13637 & 16537 & 1776 & 223916 & 33215 & 41260 & 45627 \\
Murcia & 8094 & 10909 & 12239 & 12301 & 13448 & 14954 & 17298 & 20790 & 22407 & 26292 \\
Navarra & 48265 & 62160 & 66796 & 69610 & 72297 & 73072 & 76484 & 89875 & 102184 & 122424 \\
Pais Vasco & 2729 & 4004 & 4192 & 4045 & 4393 & 5308 & 6543 & 8277 & 10085 & 11187 \\
La Rioja & 55956 & 82345 & 89294 & 93291 & 101899 & 117684 & 139506 & 181872 & 204640 & 228377 \\
Valencia & $\mathbf{5 7 9 8 3 6}$ & $\mathbf{7 7 1 2 4 5}$ & 833115 & 889991 & 969994 & 1093105 & 1296723 & 4611481 & $\mathbf{1 8 9 2 1 3 1}$ & 2123603 \\
\hline Total Nacional & & & & & & & & &
\end{tabular}

FUENTE: Instituto Nacional de Empleo

(*) Provisional 


\section{Cuadro 4}

Importe total de gastos de prestaciones por desempleo

(tasas de crecimiento)

\begin{tabular}{|lrrrrrrrrrr|}
\hline $\begin{array}{l}\text { Comunidades } \\
\text { Autónomas }\end{array}$ & $\mathbf{1 9 8 5}$ & 1986 & 1987 & 1988 & 1989 & 1990 & 1991 & 1992 & $1993^{*}$ & TMAA $^{* *}$ \\
\hline Andalucía & 25.13 & 13.78 & 14.82 & 10.55 & 15.17 & 19.88 & 20.39 & 16.28 & 7.65 & 15.85 \\
Aragón & 19.65 & 2.36 & -1.11 & 6.79 & 9.98 & 15.57 & 36.04 & 17.02 & 17.52 & 13.30 \\
Asturias & 21.50 & 3.24 & 9.90 & 8.72 & 9.06 & 12.65 & 8.61 & 19.47 & 27.88 & 13.21 \\
Baleares & 28.64 & 15.22 & 8.45 & 28.25 & 18.36 & 22.41 & 16.92 & 9.38 & -4.18 & 15.51 \\
Canarias & 56.50 & 10.89 & 19.86 & 22.73 & 21.57 & 34.65 & 26.98 & 8.84 & -6.09 & 20.67 \\
Cantabria & 36.21 & 14.57 & 8.85 & 18.26 & 1.81 & 9.96 & 28.19 & 15.48 & 21.77 & 16.83 \\
Castilla León & 9.21 & 8.87 & 8.66 & 8.53 & 9.38 & 17.30 & 25.33 & 23.05 & 8.38 & 13.01 \\
Cast. La Mancha & $\mathbf{3 1 . 2 9}$ & 15.93 & 10.89 & 12.85 & 19.59 & 15.73 & 22.79 & 24.34 & 9.48 & 17.92 \\
Cataluña & 39.66 & 6.04 & 0.70 & 4.96 & 11.63 & 21.65 & 28.90 & 16.01 & 22.67 & 16.32 \\
Extremadura & 18.72 & 20.51 & 8.81 & 10.85 & 10.88 & 16.21 & 14.57 & 13.61 & 5.07 & 13.15 \\
Galicia & 49.46 & -5.72 & 10.17 & 8.21 & 13.78 & 16.98 & 24.60 & 16.41 & 8.95 & 15.06 \\
Madrid & $\mathbf{3 5 . 3 7}$ & 3.72 & 1.31 & 4.37 & 12.73 & 17.78 & 24.58 & 25.37 & 19.06 & 15.52 \\
Murcia & 37.06 & 9.83 & 2.70 & 21.27 & 7.41 & 31.27 & 42.46 & 24.22 & 10.58 & 20.03 \\
Navarra & 34.78 & 12.19 & 0.51 & 9.32 & 11.20 & 15.67 & 20.19 & 7.78 & 17.34 & 13.99 \\
País Vasco & 28.79 & 7.46 & 4.21 & 3.86 & 1.07 & 4.67 & 17.51 & 13.70 & 19.81 & 10.90 \\
La Rioja & $\mathbf{4 6 . 7 2}$ & 4.70 & -3.51 & 8.60 & 20.83 & 23.27 & 26.50 & 21.84 & 10.93 & 16.97 \\
Valencia & 47.16 & 8.44 & 4.48 & 9.23 & 15.49 & 18.54 & 30.37 & 12.52 & 11.60 & 16.91 \\
\hline Total Nacional & $\mathbf{3 3 . 0 1}$ & $\mathbf{8 . 0 2}$ & $\mathbf{6 . 8 3}$ & $\mathbf{8 . 9 9}$ & $\mathbf{1 2 . 6 9}$ & $\mathbf{1 8 . 6 3}$ & $\mathbf{2 4 . 2 7}$ & $\mathbf{1 6 . 8 0}$ & $\mathbf{1 2 . 8 3}$ & $\mathbf{1 5 . 5 2}$ \\
\hline
\end{tabular}

Fuente: Elaboración propia. Instituto Nacional de Empleo

(*) Provisional

(**) Tasa Media Anual Acumulativa. 


\section{Cuadro 5}

Importe total de gastos de prestaciones por desempleo

(estructura porcentual)

\begin{tabular}{|lrrrrrrrrrrr|}
\hline $\begin{array}{l}\text { Comunidades } \\
\text { Autónomas }\end{array}$ & 1984 & 1985 & 1986 & 1987 & 1988 & 1989 & 1990 & 1991 & 1992 & $1993\left(^{*}\right)$ & Media \\
\hline Andalucía & 20.52 & 19.30 & 20.33 & 21.85 & 22.17 & 22.65 & 22.89 & 22.18 & 22.08 & 21.07 & 21.50 \\
Aragón & 2.90 & 2.61 & 2.48 & 2.29 & 2.24 & 2.19 & 2.13 & 2.34 & 2.34 & 2.44 & 2.40 \\
Asturias & 2.64 & 2.68 & 2.56 & 2.64 & 2.63 & 2.55 & 2.42 & 2.11 & 2.16 & 2.45 & 2.51 \\
Baleares & 1.94 & 1.87 & 2.00 & 2.03 & 2.39 & 2.51 & 2.59 & 2.43 & 2.28 & 1.94 & 2.20 \\
Canarias & 2.49 & 2.93 & 3.01 & 3.38 & 3.80 & 4.10 & 4.66 & 4.76 & 4.43 & 3.69 & 3.72 \\
Cantabria & 1.12 & 1.14 & 1.21 & 1.24 & 1.34 & 1.21 & 1.12 & 1.16 & 1.15 & 1.24 & 1.19 \\
Castilla León & 5.25 & 4.31 & 4.34 & 4.42 & 4.40 & 4.27 & 4.22 & 4.26 & 4.49 & 4.31 & 4.43 \\
Castilla La Mancha & 2.82 & 2.79 & 2.99 & 3.11 & 3.22 & 3.41 & 3.33 & 3.29 & 3.50 & 3.40 & 3.19 \\
Cataluña & 16.96 & 17.80 & 17.48 & 16.47 & 15.87 & 15.73 & 16.12 & 16.72 & 16.60 & 18.05 & 16.78 \\
Extremadura & 3.04 & 2.71 & 3.03 & 3.08 & 3.14 & 3.08 & 3.02 & 2.79 & 2.71 & 2.52 & 2.91 \\
Galicia & 5.74 & 6.45 & 5.63 & 5.81 & 5.76 & 5.82 & 5.74 & 5.75 & 5.73 & 5.54 & 5.80 \\
Madrid & 12.75 & 12.97 & 12.45 & 11.81 & 11.31 & 11.31 & 11.23 & 11.26 & 12.09 & 12.76 & 11.99 \\
Murcia & 1.52 & 1.57 & 1.59 & 1.53 & 1.70 & 1.62 & 1.80 & 2.06 & 2.19 & 2.15 & 1.77 \\
Navarra & 1.40 & 1.41 & 1.47 & 1.38 & 1.39 & 1.37 & 1.33 & 1.29 & 1.19 & 1.24 & 1.35 \\
País Vasco & 8.32 & 8.06 & 8.02 & 7.82 & 7.45 & 6.68 & 5.90 & 5.58 & 5.43 & 5.76 & 6.90 \\
La Rioja & 0.47 & 0.52 & 0.50 & 0.45 & 0.45 & 0.49 & 0.50 & 0.51 & 0.54 & 0.53 & 0.50 \\
Valencia & 9.65 & 10.68 & 10.72 & 10.48 & 10.51 & 10.77 & 10.76 & 11.29 & 10.87 & 10.75 & 10.65 \\
\hline Total Nacional & 100.00 & 100.00 & 100.00 & 100.00 & 100.00 & 100.00 & 100.00 & 100.00 & 100.00 & 100.00 & 100.00 \\
\hline
\end{tabular}

Fuente: Elaboración propia. Instituto Nacional de Empleo

(*) Provisional 


\section{Cuadro 6}

Coste medio anual de gasto de prestaciones por trabajador parado registrado en las oficinas del INEM

\begin{tabular}{|lcccccccccc|}
\hline $\begin{array}{l}\text { Comunidades } \\
\text { Autónomas }\end{array}$ & 1984 & \multirow{2}{*}{1985} & \multirow{2}{*}{1986} & 1987 & 1988 & 1989 & 1990 & 1991 & 1992 & 1993 \\
\hline Andalucia & 222933 & 262996 & 281057 & 296300 & 324782 & 398183 & 517523 & 654428 & 777170 & 778538 \\
Aragón & 262259 & 294088 & 305481 & 293396 & 326967 & 412914 & 528187 & 760626 & 966250 & 944927 \\
Asturias & 243926 & 282982 & 280644 & 279750 & 303413 & 359071 & 439048 & 491994 & 570477 & 699153 \\
Baleares & 299653 & 399309 & 420606 & 428009 & 556827 & 695838 & 812615 & 988363 & 1039201 & 911885 \\
Canarias & 140946 & 204237 & 218770 & 236250 & 283129 & 381908 & 528577 & 660071 & 743825 & 815745 \\
Cantabria & 232222 & 296141 & 322006 & 311785 & 368725 & 416730 & 518577 & 660071 & 743828 & 815745 \\
Castilla León & 236152 & 233776 & 235939 & 232561 & 253438 & 317356 & 398827 & 529961 & 665461 & 664978 \\
Castilla La Mancha & 181754 & 218496 & 235595 & 242678 & 277774 & 374538 & 465728 & 543723 & 750752 & 724558 \\
Cataluña & 220193 & 302245 & 315981 & 326691 & 390485 & 533825 & 773152 & 1001748 & 1211256 & 1200225 \\
Extremadura & 277107 & 291813 & 332213 & 312483 & 320126 & 356936 & 419123 & 508471 & 628190 & 638796 \\
Galicia & 218967 & 307447 & 267487 & 268555 & 277755 & 388372 & 413871 & 619737 & 1085410 & 1031893 \\
Madrid & 256536 & 348717 & 370057 & 373299 & 414320 & 558852 & 725428 & 878765 & 1085410 & 1031893 \\
Murcia & 179654 & 219818 & 231324 & 226153 & 280288 & 348959 & 538476 & 760069 & 856017 & 777291 \\
Navarra & 240893 & 299698 & 328123 & 321175 & 369451 & 470252 & 504825 & 787500 & 910854 & 981045 \\
País Vasco & 344258 & 410297 & 407790 & 386507 & 410312 & 469614 & 540141 & 685023 & 830764 & 917721 \\
La Rioja & 239386 & 312813 & 320000 & 313566 & 392232 & 576957 & 743523 & 890000 & 1039691 & 964397 \\
Valencia & 221257 & 294510 & 303103 & 297105 & 328495 & 429974 & 534711 & 703023 & 798439 & 787779 \\
\hline Total Nacional & $\mathbf{2 3 4 2 3 9}$ & $\mathbf{2 9 1 9 1 7}$ & $\mathbf{3 0 2 0 0 6}$ & $\mathbf{3 0 4 3 5 4}$ & 339360 & $\mathbf{4 2 8 6 1 8}$ & $\mathbf{5 5 1 7 9 7}$ & $\mathbf{7 0 4 0 1 1}$ & $\mathbf{8 3 2 8 3 8}$ & $\mathbf{8 3 6 7 8 9}$ \\
\hline
\end{tabular}

Fuente: Instituto Nacional de Empleo 


\section{Cuadro 7}

Coste medio anual del gasto de prestaciones por trabajador parado registrado en las oficinas del INEM (Tasas de crecimiento)

\begin{tabular}{|lrrrrrrrrrr|}
\hline $\begin{array}{l}\text { Comunidades } \\
\text { Autónomas }\end{array}$ & 1985 & 1986 & 1987 & 1988 & 1989 & 1990 & 1991 & 1992 & 1993 & TMAA \\
\hline Andalucia & 12.91 & 6.87 & 5.42 & 9.61 & 22.60 & 29.97 & 26.45 & 18.76 & 0.18 & 14.35 \\
Aragón & 12.14 & 3.87 & -3.96 & 11.44 & 26.29 & 27.92 & 44.01 & 27.03 & -2.21 & 15.97 \\
Asturias & 16.01 & -0.83 & -0.32 & 8.46 & 18.34 & 22.27 & 12.06 & 15.95 & 22.56 & 12.41 \\
Baleares & 33.26 & 5.33 & 1.76 & 30.10 & 24.96 & 16.78 & 21.63 & 5.14 & -12.25 & 13.16 \\
Canarias & 44.90 & 7.12 & 7.99 & 19.84 & 34.89 & 37.58 & 23.96 & 2.57 & -12.08 & 17.19 \\
Cantabria & 27.52 & 8.73 & -3.17 & 18.26 & 13.02 & 24.44 & 27.29 & 12.69 & 9.67 & 14.98 \\
Castilla León & -1.01 & 0.93 & -1.43 & 8.98 & 25.22 & 25.67 & 32.88 & 25.57 & -0.07 & 12.19 \\
Castilla La Mancha & 20.22 & 7.83 & 3.01 & 14.46 & 34.84 & 24.35 & 16.75 & 38.08 & -3.49 & 16.61 \\
Cataluña & 37.26 & 4.54 & 3.39 & 19.53 & 36.71 & 44.83 & 29.57 & 20.91 & -0.91 & 20.73 \\
Extremadura & 5.31 & 13.84 & -5.94 & 2.45 & 11.50 & 17.42 & 21.32 & 23.54 & 1.69 & 9.72 \\
Galicia & 40.41 & -13.00 & 0.40 & 3.43 & 39.83 & 6.57 & 25.58 & 16.34 & 1.77 & 12.17 \\
Madrid & 35.93 & 6.12 & 0.88 & 10.99 & 34.88 & 29.81 & 21.14 & 23.52 & -4.93 & 16.73 \\
Murcia & 22.36 & 5.23 & -2.24 & 23.94 & 24.50 & 54.31 & 41.15 & 12.62 & -9.20 & 17.67 \\
Navarra & 24.41 & 9.48 & -2.12 & 15.03 & 27.28 & 7.35 & 55.99 & 15.66 & 7.71 & 16.89 \\
Pais Vasco & 19.18 & -0.61 & -5.22 & 6.16 & 14.45 & 15.02 & 26.82 & 21.28 & 10.47 & 11.51 \\
La Rioja & 30.67 & 2.30 & -2.01 & 25.09 & 47.10 & 28.87 & 19.70 & 16.82 & -7.24 & 16.75 \\
Valencia & 33.11 & 2.92 & -1.98 & 10.57 & 30.89 & 24.36 & 31.48 & 13.57 & -1.34 & 15.15 \\
\hline TOTAL NACIONAL & 24.62 & 3.46 & 0.78 & 11.50 & 26.30 & 28.74 & 27.59 & 18.30 & 0.47 & 15.20 \\
\hline
\end{tabular}

Fuente: Instituto Nacional de Empleo. Elaboración propia. 


\section{Cuadro 8}

Coste medio anual del gasto de prestaciones por trabajador parado registrado en las oficinas del INEM

(Estructura porcentual)

\begin{tabular}{|lrrrrrrrrrrr|}
\hline $\begin{array}{l}\text { Comunidades } \\
\text { Autónomas }\end{array}$ & 1984 & 1985 & \multicolumn{1}{c}{1986} & 1987 & 1988 & 1989 & 1990 & 1991 & 1992 & 1993 & Media \\
\hline Andalucia & 99.44 & 90.09 & 93.06 & 97.35 & 95.70 & 92.90 & 93.79 & 92.96 & 93.32 & 93.04 & 94.17 \\
Aragón & 111.96 & 100.74 & 101.15 & 96.40 & 96.35 & 96.34 & 95.72 & 108.04 & 116.02 & 112.92 & 103.56 \\
Asturias & 104.14 & 96.94 & 92.93 & 91.92 & 89.41 & 83.77 & 79.57 & 69.88 & 68.50 & 83.55 & 86.06 \\
Baleares & 127.93 & 136.79 & 139.27 & 140.63 & 164.08 & 162.34 & 147.27 & 140.39 & 124.78 & 108.97 & 139.25 \\
Canarias & 60.17 & 69.96 & 72.44 & 77.62 & 83.43 & 89.10 & 95.22 & 92.52 & 80.21 & 70.19 & 79.09 \\
Cantabria & 99.14 & 101.45 & 106.62 & 102.44 & 108.65 & 97.23 & 93.98 & 93.76 & 89.31 & 97.49 & 99.01 \\
Castilla León & 100.82 & 80.08 & 78.12 & 76.41 & 74.68 & 74.04 & 72.28 & 75.28 & 90.14 & 86.59 & 81.78 \\
Castilla La Mancha & 77.59 & 74.85 & 78.01 & 79.74 & 81.85 & 87.38 & 84.40 & 77.23 & 90.14 & 86.59 & 81.78 \\
Cataluña & 94.00 & 103.54 & 104.63 & 107.34 & 115.07 & 124.55 & 140.12 & 142.29 & 145.44 & 143.43 & 122.04 \\
Extremadura & 118.30 & 99.96 & 110.00 & 102.67 & 94.33 & 83.28 & 75.93 & 72.22 & 75.43 & 76.34 & 90.85 \\
Galicia & 93.48 & 105.32 & 88.57 & 88.24 & 81.85 & 90.61 & 75.00 & 73.83 & 72.60 & 73.54 & 84.30 \\
Madrid & 109.52 & 119.46 & 122.53 & 122.65 & 122.09 & 130.38 & 131.47 & 124.83 & 130.33 & 123.32 & 123.66 \\
Murcia & 76.70 & 75.30 & 76.60 & 74.31 & 82.59 & 81.41 & 97.59 & 107.96 & 102.78 & 92.89 & 86.81 \\
Navarra & 102.84 & 102.67 & 108.65 & 105.53 & 108.87 & 109.71 & 91.49 & 111.86 & 109.37 & 117.24 & 106.82 \\
País Vasco & 146.97 & 140.55 & 135.03 & 126.99 & 120.91 & 109.56 & 97.89 & 97.30 & 99.75 & 109.67 & 118.46 \\
La Rioja & 102.20 & 107.16 & 105.96 & 103.03 & 115.58 & 134.61 & 134.75 & 126.42 & 124.84 & 115.25 & 116.98 \\
Valencia & 94.46 & 100.89 & 100.36 & 97.62 & 96.80 & 100.32 & 96.90 & 99.86 & 95.87 & 94.14 & 97.72 \\
\hline Total Nacional & 100.00 & 100.00 & 100.00 & 100.00 & 100.00 & 100.00 & 100.00 & 100.00 & 100.00 & 100.00 & 100.00 \\
\hline
\end{tabular}

Fuente: Instituto Nacional de Empleo. Elaboración propia. 


\section{Cuadro 9}

Posiciones relativas en la percepción de subsidios de las comunidades autónomas según costes de prestaciones

\begin{tabular}{|lcccc|}
\hline COMUNIDADES & $\begin{array}{c}\text { Magnitud } \\
\text { Costes } \\
\text { totales }\end{array}$ & $\begin{array}{c}\text { Crecimiento } \\
\text { Costes } \\
\text { totales }\end{array}$ & $\begin{array}{c}\text { Magnitud } \\
\text { Costes } \\
\text { unitarios }\end{array}$ & $\begin{array}{c}\text { Crecimiento } \\
\text { Costes } \\
\text { unitarios }\end{array}$ \\
\hline Andalucía & 1 & 8 & 10 & 11 \\
Aragón & 12 & 13 & 7 & 8 \\
Asturias & 11 & 14 & 13 & 13 \\
Baleares & 13 & 10 & 1 & 12 \\
Canarias & 8 & 1 & 17 & 3 \\
Cantabria & 16 & 6 & 8 & 10 \\
Castilla León & 7 & 16 & 16 & 14 \\
Castilla La Mancha & 9 & 3 & 15 & 7 \\
Cataluña & 2 & 7 & 3 & 1 \\
Extremadura & 10 & 15 & 11 & 17 \\
Galicia & 6 & 11 & 14 & 15 \\
Madrid & 3 & 9 & 2 & 6 \\
Murcia & 14 & 2 & 12 & 2 \\
Navarra & 15 & 12 & 6 & 4 \\
País Vasco & 5 & 17 & 4 & 16 \\
La Rioja & 17 & 4 & 5 & 5 \\
Valencia & 4 & 5 & 9 & 9 \\
\hline
\end{tabular}

Fuente: Instituto Nacional de Empleo.

Elaboración propia. 